\title{
Prevalencia de depresión en niños escolarizados entre 8 y 12 años del oriente antioqueño a partir del "child depression inventory" -CDI-
}

\author{
Stefano Vinaccia* \\ Ana Milena Gaviria \\ Luis Federico Atehortúa \\ Piedad Helena Martínez \\ Catalina Trujillo \\ Japcy Margarita Quiceno \\ Universidad de San Buenaventura, \\ Medellín, Colombia
}

Recibido: abril 24 de 2006 Revisado: mayo 15 de 2006 Aceptado: junio 9 de 2006

\section{Resumen}

El objetivo de la siguiente investigación fue estudiar la prevalencia de la depresión en una población infantil del oriente antioqueño. Muestra: 768 sujetos entre los 8 y los 12 años, de los cuales 379 eran de género masculino y 389 de género femenino residentes en el municipio de La Ceja (Antioquia). Los instrumentos que se utilizaron fueron Children Depresión Inventory (CDI) versión en español de la escala original de Kovacs (1992) de Davanzo y cols. (2004). Se encontró una media de depresión en la escala total de 10,52 y una desvaloración estándar de 5,61. La prevalencia de sintomatología depresiva encontrada fue de un $25.2 \%$ de la muestra investigada utilizando como punto de corte puntajes superiores a los 17 puntos. Esto evidencia que es alta la sintomatología depresiva encontrada en niños escolares del oriente antioqueño; se necesita mayor atención e investigación en esta población.

Palabras clave: CDI, depresión infantil, Antioquia, Colombia.

* Correspondencia: Stefano Vinaccia. Universidad de San Buenaventura, Medellín. Apartado Aéreo:81240 - Envigado- Antioquia. Correo electrónico: vinalpi47@hotmail.com 


\section{Abstract}

The objective on this investigation was to study the prevalence of depression on children from east of Antioquia. Sample: 768 subjects between 8 and 12 years; 379 of them were boys, and 389 were girls; all of them were residents of La Ceja (Antioquia). Implement: Children Depression Inventory (CDI) Spanish Version of Kovacs' original scale (1992) of Davanzo and cols (2004). Results: the MD of depression's total scale was of 10.52 and SD was of $(5,61)$. The prevalence of depressive symptoms was of $25.2 \%$ of the sample. This was concluded by establishing superior scores of 17 points as the cut point. Conclusions: There are high depressive symptoms on children from east of Antioquia. There is a need of attention and research to this population.

Key words: CDI, child depression, Antioquia, Colombia.

\section{Introducción}

En los últimos años, numerosas investigaciones han centrado su atención en aspectos asociados con la salud mental de los niños y de los adolescentes estudiándose aspectos demográficos, epidemiológicos y de carga de la enfermedad (Gómez, 2005). La magnitud de los trastornos mentales en niños y adolescentes puede apreciarse en algunos datos tomados del reporte de la Organización Mundial de la Salud (OMS), donde se plantea que el $20 \%$ de los niños y adolescentes sufre de alguna enfermedad mental (Gómez, 2005; OMS,2003).

En Colombia, los resultados preliminares de la investigación del "estudio nacional de salud mental y estilos de vida" desarrollado por el Ministerio de protección social (2004). plantearon que el $40.1 \%$ de la población adulta a tenido un trastorno mental en su vida.

En la población masculina, los primeros tipos de trastornos fueron: abuso de alcohol, fobias y tras- tornos de conducta; en la población femenina: depresión y fobias.

La depresión como trastorno de estado de ánimo según un informe de la OMS (2003) aparece como la principal causa de discapacidad y la cuarta de las 10 causas principales de la carga de morbilidad en el ámbito mundial. Se pronostica que para el año 2020 este trastorno ocupará el $2^{\circ}$ lugar entre los trastornos mentales y físicos (OMS, 2003; Gómez, Álviz y Sepúlveda, 2003).

Torres y Montoya (1997) mostraron en un estudio de salud mental cómo el $13,3 \%$ de pre-adolescentes y adolescentes entre 12 y 15 años había sufrido síntomas depresivos y que ese porcentaje aumentaba al $20.1 \%$ entre los jóvenes entre los 16 y los 19 años. Adicionalmente, es bien sabido que la depresión infantil ha tenido serios problemas por ser aceptada por la comunidad científica (Del Barrio y cols., 2002).

Ahora bien, en un espacio relativamente corto, en la década de los sesenta, se pasó de la opinión 
generalizada de negar la depresión infantil, a aceptar su existencia y a la discusión de "en qué consiste y cuáles son sus características"(Del Barrio y cols., 2002).

Por otro lado, Riso (1992) plantea que la baja autoestima, cambios de sueño, aislamiento social, cambios del apetito y del peso, hiperactividad, disforia e ideación suicida son sus síntomas fundamentales. Hoy existe una pluralidad de instrumentos para la evaluación de la depresión infantil. Entre los muchos instrumentos existentes se destaca el CDI (Childrens Depresión Inventory) por su difusión y uso en distintos países.

Al respecto, Davanzo y cols. (2004) validaron al español las versiones larga y corta del CDI con una muestra de 205 estudiantes latinos de diferentes países de Centroamérica y Suramérica, encontrando un buen índice de consistencia interna en la prueba (alfa de Cronbach de .85) y un $11.2 \%$ de sujetos a riesgo de depresión.

En Colombia, Mantilla y cols. (2003) investigaron con la versión corta del CDI de 10 ítems una muestra de 239 niños escolarizados de clase alta y baja de la ciudad de Bucaramanga. Se encontró que el 9.2\% se encontraba deprimido; llegando a la conclusión de que la sintomatología depresiva aumentaba con la edad y el grado de escolaridad.

En el departamento de Antioquia (Colombia), Gómez, Alviz y Sepúlveda (2003) con la versión larga del CDI de 27 ítems desarrollaron una investigación en la ciudad de Medellín con una muestra de 207 niños y niñas entre 8 y 12 años de estratos 3 , 4 y 5 . Encontrándose promedios más bajos de depresión en comparación con las medias encontradas en Estados Unidos por Kovacs (1992), la autora original de la prueba.

El objetivo de este estudio descriptivo mediante encuestas de tipo transversal (Montero y León, 2005) fue estudiar la prevalencia de depresión en una muestra de niños y niñas de sectores urbanos del municipio La Ceja ubicado en el oriente Antioqueño.

\section{Método}

\section{Participantes}

La población está comprendida por niños y niñas entre los 8 y 12 años de edad del área urbana del municipio de La Ceja, Antioquia, que se encuentran escolarizados en los grados $3^{\circ}, 4^{\circ}, 5^{\circ}, 6^{\circ} \mathrm{y}$ $7^{\circ}$ de los diferentes colegios públicos y privados del municipio, sin características clínicas detectadas frente a la depresión. El tamaño de la muestra fue de 768 personas.

Para el cálculo del tamaño de la muestra se utilizó el programa STATCALC que viene con el paquete Epi- Info.

Los requerimientos de información fueron:

- Tamaño poblacional: 9,554

- Prevalencia esperada: $2 \%^{1}$

- Peor resultado esperado: $1 \%$

- Nivel de confianza: $95 \%$

Total: 698.

Más 10\% de margen de error: 768 muestras

La fórmula utilizada para este cálculo fue:

$$
S=Z^{*} Z(P(1-P)) /\left(D^{*} D\right)
$$

Donde: $\mathrm{d}=$ mitad del ancho del intervalo de prevalencia que nos interesa estudiar (confianza muestral).

$\mathrm{P}=$ prevalencia esperada del evento, $\mathrm{Z}$ = percentil de la distribución normal estándar determinado por el nivel de confianza especificado; esto es, 1.96 para el nivel de confianza del $95 \%$.

S se ajusta para la población finita por un factor de corrección para obtener el tamaño muestral estimado, como sigue:

Tamaño muestral $=\mathrm{S} /(1+(\mathrm{S} /$ población $))$ 


\section{Instrumento}

“Children's Depression Inventory" -CDI-, (Inventario de Depression Infantil), diseñado originalmente por Kovacs (1992) en Estados Unidos, en la versión en español de Davanzo y cols. (2004).

Este inventario consta de 27 preguntas con un formato tipo likert con tres posibilidades de respuesta, 0 ausencia de síntomas, 1 síntomas moderados, 2 síntomas severos. El coeficiente Alfa de Cronbach encontrado en la validación del instrumento fue de .85 .

\section{Procedimiento}

Las pruebas se aplicaron de forma colectiva a los 768 niños y niñas. Para ello, se realizó una jornada lúdica y educativa en donde una vez diligenciaron las pruebas se les brindó información sobre el objetivo del estudio. Antes de aplicar las pruebas se les explicó en qué consistía y para qué se iban a emplear los resultados; una vez se contó con el consentimiento informado de los niños, se procedió al autodiligenciamiento de los cuestionarios, brindando la asesoría y colaboración necesaria.

\section{Resultados}

Tabla 1. Estadísticas de las variables sociodemográficas

\begin{tabular}{|l|cccc|}
\hline & Media & Moda & Mínimo & Máximo \\
\hline Edad & 10,04 & 11 & 8 & 13 \\
Sexo & 1,51 & 2 & 1 & 2 \\
\hline
\end{tabular}

La tabla 1 presenta las variables sociodemográficas; en ella se puede observar que la variable edad está comprendida entre 8 y 13 años, siendo los estudiantes de 11 años el grupo más sobresaliente. El género femenino, como se muestra, es el más destacado dentro del grupo estudiado.
Tabla 2. Frecuencias de la variable edad en años cumplidos

\begin{tabular}{|c|ccc|}
\hline & Frecuencia & $\begin{array}{c}\text { Porcentaje } \\
\text { absoluto }\end{array}$ & $\begin{array}{c}\text { Porcentaje } \\
\text { acumulado }\end{array}$ \\
\hline 8 & 113 & 14,7 & 14,7 \\
9 & 146 & 19,0 & 33,7 \\
10 & 185 & 24,1 & 57,8 \\
11 & 247 & 32,2 & 90,0 \\
12 & 76 & 9,9 & 99,9 \\
13 & 1 &, 1 & 100,0 \\
Total & 768 & 100,0 & \\
\hline
\end{tabular}

Se observa en la tabla 2 que la población está muy dispersa en cuanto a la variable edad en años cumplidos, aunque se hace notorio que la mayoría de la población se ubica en el grupo de niños con 11 años y de esta edad para abajo, es decir el 90,0\% de la población, pertenecen a una edad comprendida entre 8 y 11 años.

Tabla 3. Frecuencias de la variable género

\begin{tabular}{|c|ccc|}
\hline & Frecuencia & $\begin{array}{c}\text { Porcentaje } \\
\text { absoluto }\end{array}$ & $\begin{array}{c}\text { Porcentaje } \\
\text { acumulado }\end{array}$ \\
\hline Masculino & 379 & 49,3 & 49,3 \\
Femenino & 389 & 50,7 & 100,0 \\
Total & 768 & 100,0 & \\
\hline
\end{tabular}

En relación con el género vemos en la tabla 3, que el $50,7 \%$ de la población pertenece al género femenino, mientras el otro $49,3 \%$ pertenece al género masculino.

Tabla 4. Análisis de fiabilidad del estudio Número de casos 27

\section{Estadísticas del estudio}

\begin{tabular}{|ccc|}
\hline Media & Varianza & $\begin{array}{c}\text { Desviación } \\
\text { estándar }\end{array}$ \\
\hline 10,52 & 31,546 & 5,617 \\
\hline
\end{tabular}


Análisis de varianza

\begin{tabular}{|c|c|c|c|c|c|c|}
\hline & Media & hínimo & Máximo & Rango & $\begin{array}{l}\text { Máximo / } \\
\text { Mínimo }\end{array}$ & Varianza \\
\hline Media Ítem & ,390 & ,057 & 1,240 & 1,182 & 21,636 & 071 \\
\hline Varianza Item & ,302 & ,078 & ,643 &, 566 & 8,293 &, 025 \\
\hline $\begin{array}{l}\text { Correlación Inter- } \\
\text { Item }\end{array}$ & 123 & 年, 129 & 319 & ,448 & $-2,467$ & , 006 \\
\hline
\end{tabular}

Gran Media 0,39

\begin{tabular}{|l|ccccc|}
\hline & Suma de Cuadrados & df & Media Cuadrados & $F$ & Sig \\
\hline Entre Individuos & 896,133 & 767 & 1,168 & & \\
Entre Items & 1411,984 & 26 & 54,307 & 201,755 &, 000 \\
Residuales & 5367,867 & 19942 &, 269 & & \\
Total & 6779,852 & 19968 &, 340 & & \\
Total & 7675,984 & 20735 &, 370 & & \\
\hline
\end{tabular}

En la tabla 4 se observa que la media y la desviación estandar encontrada en el estudio fueron respectivamente de 10.52 y 5,61. Comparando nuestros resultados con la investigación de Gómez, Alviz y Sepúlveda (2003) con una muestra de la ciudad de Medellín que tuvieron una media de 7,94 y una DS de 5.2 nuestros resultados son mucho más elevados en promedios de depresión; lo mismo podemos decir en relación con las investigaciones de Davanzo y cols. (2004) con hispanos residentes en Estados Unidos (media 8.8 y DS de 6.6 y la investigación Americana original de Kovac (1992) con una media de 9,98 . Por el contrario, se encontraron resultados similares a la investigación de $\mathrm{Del} \mathrm{Ba}$ rrio y Mestre (1989) en España con medias de varones de $10.2(5.08)$ y de niñas de $10.76(5.48)$.

Tabla 5. Análisis de fiabilidad para cada factor

\begin{tabular}{|c|c|c|c|c|}
\hline & $\begin{array}{c}\text { Escala } \\
\text { de Media }\end{array}$ & $\begin{array}{c}\text { Escala } \\
\text { de Varianza }\end{array}$ & $\begin{array}{c}\text { Correlación } \\
\text { Total }\end{array}$ & $\begin{array}{c}\text { Alfa de } \\
\text { Cronbach }\end{array}$ \\
\hline Item $\mathrm{N}^{\circ} 1$ & 10,40 & 29,993 & ,341 & ,762 \\
\hline Item $\mathrm{N}^{*} 2$ & 9,96 & 29,471 & ,311 & ,762 \\
\hline Item $\mathrm{N}^{*} 3$ & 10,36 & 29,737 &, 382 &, 760 \\
\hline Item $\mathrm{N}^{\circ} 4$ & 10,06 & 30,158 & 197 &, 768 \\
\hline Item $\mathrm{N}^{*} 5$ & 10,47 & 30,750 & ,233 &, 766 \\
\hline Item $N^{*} 6$ & 9,86 & 30,757 &, 084 &, 774 \\
\hline Item $\mathrm{N}^{\circ} 7$ & 10,44 & 30,085 & 391 & 761 \\
\hline Item $\mathrm{N}^{-} 8$ & 10,15 & 29,442 & ,295 &, 763 \\
\hline Item $N^{*} 9$ & 10,14 & 29,574 &, 296 &, 763 \\
\hline Item $\mathrm{N}^{\circ} 10$ & 10,41 & 29,744 & ,346 &, 761 \\
\hline Item $\mathrm{N}^{-} 11$ & 9,98 & 28,331 & ,308 &, 763 \\
\hline Item $\mathrm{N}^{\circ} 12$ & 10,35 & 30,184 &, 253 &, 765 \\
\hline Item $\mathbf{N}^{*} 13$ & 9,98 & 28,761 & ,321 &, 761 \\
\hline item $\mathrm{N}^{*} 14$ & 10,17 & 28,959 &, 421 &, 756 \\
\hline Item $\mathrm{N}^{\circ} 15$ & 9,63 & 29,008 &, 219 &, 770 \\
\hline Item $\mathrm{N}^{*} 16$ & 10,09 & 28,543 & ,344 &, 760 \\
\hline Item $N^{*} 17$ & 10,41 & 30,177 &, 310 &, 763 \\
\hline Item $\mathrm{N}^{\circ} 18$ & 10,02 & 28,520 &, 285 &, 765 \\
\hline Item N" 19 & 9,28 & 31,544 & -,061 &, 786 \\
\hline Item $\mathrm{N}^{*} 20$ & 10,25 & 29,130 &, 404 &, 757 \\
\hline Item $\mathrm{N}^{\circ} 21$ & 10,19 & 28,648 & ,443 & ,754 \\
\hline Item $\mathrm{N}^{-} 22$ & 10,15 & 29,207 & ,355 &, 759 \\
\hline Item $\mathrm{N}^{*} 23$ & 10,22 & 28,887 & ,399 &, 757 \\
\hline Item $\mathrm{N}^{\circ} 24$ & 9,94 & 28,191 &, 437 &, 754 \\
\hline Item $\mathrm{N}^{-2} 25$ & 10,35 & 29,691 & ,356 &, 760 \\
\hline Item $\mathrm{N}^{\circ} 26$ & 10,03 & 29,476 &, 286 &, 763 \\
\hline Item $\mathrm{N}^{\circ} 27$ & 10,35 & 29,846 & 339 &, 761 \\
\hline
\end{tabular}

Alfa de Crobach: 0,70 Ítems estandarizados: 0,791 
Se observa en la tabla 5 que para cada factor o ítem estudiado, el valor de alfa es superior a 0,5 , lo que indica que el criterio de aceptación dentro del estudio es bueno. Estos resultados confirman la fiabilidad de la prueba en diferentes contextos culturales de Estados Unidos, América Latina, Asia, y Europa con Alfas de Cronbach entre 0.79 y 0.94 .

Tabla 6. Prevalencia de la depresión

\begin{tabular}{|c|c|c|c|c|}
\hline & & \multicolumn{2}{|c|}{ Sexo } & \multirow[b]{2}{*}{ Total } \\
\hline & & Masculino & Femenino & \\
\hline \multirow[t]{2}{*}{17} & Cuenta & 8 & 9 & 17 \\
\hline & \% Sexo & $2,1 \%$ & $2,3 \%$ & $2,2 \%$ \\
\hline \multirow[t]{2}{*}{18} & Cuenta & 6 & 4 & 10 \\
\hline & \% Sexo & $1,6 \%$ & $1,0 \%$ & $1,3 \%$ \\
\hline \multirow[t]{2}{*}{19} & Cuenta & 6 & 6 & 12 \\
\hline & $\%$ Sexo & $1,6 \%$ & $1,5 \%$ & $1,6 \%$ \\
\hline \multirow[t]{2}{*}{20} & Cuenta & 6 & 9 & 15 \\
\hline & \% Sexo & $1,6 \%$ & $2,3 \%$ & $2,0 \%$ \\
\hline \multirow[t]{2}{*}{21} & Cuenta & 8 & 3 & 11 \\
\hline & \% Sexo & $2,1 \%$ &, $8 \%$ & $1,4 \%$ \\
\hline \multirow[t]{2}{*}{22} & Cuenta & 3 & 4 & 7 \\
\hline & \% Sexo & ,8\% & $1,0 \%$ & ,9\% \\
\hline \multirow[t]{2}{*}{23} & Cuenta & 1 & 0 & 1 \\
\hline & \% Sexo &, $3 \%$ &, $0 \%$ &, $1 \%$ \\
\hline \multirow[t]{2}{*}{24} & Cuenta & 4 & 2 & 6 \\
\hline & \% Sexo & $1,1 \%$ &, $5 \%$ &, $8 \%$ \\
\hline \multirow[t]{2}{*}{25} & Cuenta & 1 & 2 & 3 \\
\hline & $\%$ Sexo &, $3 \%$ &, $5 \%$ &, $4 \%$ \\
\hline \multirow[t]{2}{*}{27} & Cuenta & 2 & 0 & 2 \\
\hline & \% Sexo &, $5 \%$ &, $0 \%$ & ,3\% \\
\hline \multirow[t]{2}{*}{28} & Cuenta & 2 & 0 & 2 \\
\hline & $\%$ Sexo &, $5 \%$ &, $0 \%$ &, $3 \%$ \\
\hline \multirow[t]{2}{*}{29} & Cuent & 1 & 1 & 2 \\
\hline & \% Sexo & , 3\% &, $3 \%$ &, $3 \%$ \\
\hline \multirow[t]{2}{*}{30} & Cuenta & 2 & 1 & 3 \\
\hline & $\%$ Sexo &, $5 \%$ &, $3 \%$ &, $4 \%$ \\
\hline \multirow[t]{2}{*}{31} & Cuenta & 3 & 0 & 3 \\
\hline & \% Sexo & ,8\% &, $0 \%$ &, $4 \%$ \\
\hline \multirow[t]{2}{*}{36} & Cuenta & 1 & 0 & 1 \\
\hline & \% Sexo & , 3\% &, $0 \%$ &, $1 \%$ \\
\hline \multirow[t]{2}{*}{37} & Cuenta & 0 & 1 & 1 \\
\hline & \% Sexo &, $0 \%$ &, $3 \%$ &, $1 \%$ \\
\hline \multirow[t]{2}{*}{ Total } & Cuenta & 54 & 42 & 96 \\
\hline & $\%$ Sexo & $14.4 \%$ & $10.8 \%$ & $25.2 \%$ \\
\hline
\end{tabular}


En relación con la tabla 6 , se asumieron dos puntos de corte para la presencia de depresión en niños. Uno de 17 puntos, estimado por Doménech y cols (1995) y por Craighead y cols. (1995) en donde aconsejan usarlo tanto en niños como en adolescentes; y otro, de 19 puntos estimado por Kovacs (1992), autora del instrumento. Teniendo en cuenta estos datos, se obtuvo una prevalencia de puntuaciones en la escala por encima de 17 puntos de $25.2 \%$, valor altamente significativo, pues la mayoría de estudios revisados presentan una prevalencia mucho menor (Davanzo y cols. 2004; Sepúlveda y cols. 2003 ); por otro lado, este porcentaje seguiría alto, $(21,7 \%)$, si el punto de corte fuera igual o mayor de 19 puntos, según el criterio expresado por Kovacs (1992).

\section{Discusión}

Luego de aplicado el instrumento y analizada la información contenida en la base de datos, se puede visualizar el comportamiento de las variables así:

En cuanto a las variables sociodemográficas, se observa que la mayoría de la población se ubica en el grupo de niños con 11 años. El 90\% de los sujetos que conformaron la muestra osciló en edades entre los 8 y 11 años.

En cuanto al género, se observa que el $50,7 \%$ de la población pertenece al género femenino, el otro $49,3 \%$ al género masculino.

En cuanto a los valores de la prevalencia de depresión, se asumieron dos puntos de corte para la presencia de depresión en niños. Uno de 17 puntos, estimado por Doménech y cols. (1995) y por Craighead y cols. (1995) en donde aconsejan usarlo tanto en niños como en adolescentes, y otro de 19 puntos estimado por Kovacs (1992). Teniendo en cuenta estos datos, se obtuvo una prevalencia de puntuaciones en la escala por encima de 17 puntos de $25.2 \%$, valor altamente significativo, pues la mayoría de estudios revisados presenta una prevalencia menor.
Los porcentajes hallados en el grupo de niños y niñas de la población general están por encima de los valores reportados por otros estudios. Los datos que se encuentran en la literatura sobre la prevalencia de la depresión en niños indican que en la población normal la prevalencia del trastorno se encuentra alrededor del 2\% (Kashani y Schmid, 1995; OMS, 2003) y en la población clínica está entre 7 y $34 \%$ (Davanzo y cols. 2004); además, comparando nuestros resultados con el estudio realizado con la población infantil normal Alemana (Gómez, Álviz y Sepúlveda, 2003), que fue del $17,5 \%$, se encuentra que el porcentaje nuestro es mucho mayor que el de los estudios realizados, significando esto que los niños y niñas de la población estudiada presenta un alto índice de sintomatología depresiva.

Por otro lado, discriminando los resultados por sexo, encontramos que, a diferencia de otras investigaciones, los hombres presentaron una prevalencia mayor de depresión (14.4\%), en comparación con las mujeres (10.8\%). Sin embargo, no es posible dejar de señalar que estas puntuaciones sobrepasan las presentadas por otros autores; con los datos obtenidos se evidencia que existe mayor prevalencia en los niños que en las niñas de este estudio. En los estudios realizados con poblaciones en los estudios realizados con poblaciones alemana, americana y española, se observa que las niñas tienden a tener puntuaciones más altas, mientras que en los niños las puntuaciones fueron menores, datos que difieren considerablemente con los resultados obtenidos en el presente estudio, en donde los niños presentan puntuaciones mayores en relación con las niñas.

Es habitual oír que hay un mayor número de depresión en las mujeres que en los hombres. Las explicaciones son múltiples y se pueden categorizar en varios aspectos biopsicosociales. Se pensó, desde una perspectiva social, que esto estaba ligado funcionalmente a los papeles del sexo o género.

Curiosamente los estudios actuales demuestran que no existe diferenciación en la aparición de sínto- 
mas de depresión entre niños y niñas antes de cumplir los 12 años. A partir de esa edad comienzan a ser más frecuentes en las niñas e incluso va incrementando a medida que aumenta la edad (adolescencia), lo que apunta a una dimensión fuertemente social de género. El sexo femenino es uno de los más potentes predictores de desarrollar una depresión en el futuro junto con problemas de interiorización (ansiedad, pensamientos suicidas y depresión), debido a que son ellas las que acuden con mayor frecuencia a ayudas psicológicas. Es de esperar entonces que el $10.8 \%$ de las niñas tengan un riesgo mayor de desarrollar trastornos del estado de animo en las siguientes etapas del desarrollo.

El hecho de que el $25.2 \%$ de los menores del municipio de La Ceja posea depresión significa que es una población a riesgo, ya que esta enfermedad produce una alta morbi-mortalidad por el riesgo suicida, conductas autolesivas, abuso de sustancias y comportamiento disocial que conlleva; además, experimentan un alto grado de sufrimiento subjetivo explicado por la presencia en el $25.2 \%$ de los menores con síntomas tales como el ánimo negativo, la anhedonia, la percepción de ineficacia, los problemas interpersonales y la autoestima negativa.

En relación con el ánimo negativo, entendido como el estado afectivo caracterizado por sensaciones de estados emocionales aversivos, como nerviosismo, miedo, disgusto, culpa e ira, y en bajo porcentaje definido como un estado de calma y serenidad, aunque no necesariamente felicidad (APA, 1994), se puede afirmar como producto de este estudio que las puntuaciones promedio obtenidas fueron: tristeza 10,7\% de la población; algo malo va a pasar 39,7\%; culpa 33,9\%; ganas de llorar 5,8\%; molestia frente a las cosas $35,2 \%$ y tomar decisiones $44 \%$.

A la hora de evaluar la anhedonia como síntoma depresivo en la población, encontramos que el $45.4 \%$ de los sujetos evaluados describe no poder divertirse con muchas cosas, el $33.4 \%$ expresa tener problemas con el sueño; mientras que al $9.8 \%$ se siente cansado siempre. El $31.5 \%$ comenta no tener buen apetito la mayoría del tiempo y al $52.3 \%$ le preocupa el dolor y la enfermedad. Estos resultados muestran que aunque hay bajos niveles de anhedonia física en la población evaluada, es importante prestarle atención al porcentaje de los niños y niñas que presentan síntomas depresivos.

Así mismo, el $26.4 \%$ dice sentirse solo siempre, el $29.3 \%$ expresa no poder divertirse en el colegio frecuentemente y el $34.8 \%$ manifiesta no tener amigos. Esto resultados evidencian una fuerte desmotivación por los contactos sociales en la población, ésta es una característica que no contribuye a proteger a los individuos de sufrir estados depresivos, pues se sabe que el contacto social sirve como refuerzo de los logros personales y medio para consolidar un buen desarrollo de la identidad en esta etapa evolutiva.

La anhedonia es definida como la pérdida notoria de la capacidad para experimentar placer de actividades que habitualmente producen sensaciones o sentimientos agradables; la incapacidad de obtener placer con objetos o situaciones físicas se denomina anhedonia física; o a través de la interacción con otras personas, anhedonia social (APA, 1994).

Kashani y Schmid (1995) definen la inefectividad como el desempeño incompetente en áreas de logro (académicas, social, o personal) o actividades dirigidas a una meta. Dentro de los ítems que componen esta variable se encuentran: hacer las cosas bien, dentro del estudio se visualiza que una minoría de la población $(15,8 \%)$ obtuvo puntajes altos, lo cual significa que los menores evaluados se ven afectados negativamente por las críticas que los demás puedan hacer a su desempeño. $\mathrm{El}$ $38 \%$ de la población expresa tener dificultades a la hora de hacer las tareas; el 36,3\% de la población expresa sentirse insatisfecho con su desempeño académico y el $8.1 \%$ de los menores manifiesta sentirse "malo" o ineficaz como los demás compañeros. Lo anterior indica que no existen problemas en el desempeño de la población en estudio.

Dentro de la variable inefectivadad uno de los ítems evaluados fue el rendimiento escolar; y aunque con 
frecuencia rendimiento escolar y depresión se encuentran unidos, esta unión no esta realmente clara. Muchos estudios empíricos avalan que la depresión es la causa del descenso y no el descenso como causa de depresión.

Doménech y cols. (1995) han encontrado que el disgusto con las propias calificaciones escolares es un fuerte predictor del desencadenamiento de una depresión futura. Es decir, la depresión puede ser causa y consecuencia de un deficiente rendimiento escolar según las circunstancias.

Los estudios muestran que los niños deprimidos presentan dificultades para atender en clase, para ejecutar tareas de clase y presentan más muestras de fatiga y tristeza que sus compañeros no deprimidos. Por otra parte, los niños y adolescentes deprimidos informan de un descenso significativamente mayor que el de los niños no deprimidos.

Para Craighead y cols. (1995), existen problemas interpersonales si el sujeto experimenta una fuerte incomodidad subjetiva en determinadas situaciones sociales, si evita ciertas interacciones, o si no es capaz de obtener los resultados deseados y/ o culturalmente aceptables, de las relaciones sociales de una manera que otras personas relevantes juzgan como apropiada. Dentro de la evaluación de esta característica en la población, entre los ítems que miden la variable "problemas interpersonales" se encuentran: obediencia, en donde se observa que la población de estudio presentó puntuaciones bajas, ya que el $4,6 \%$ se ubica en la categoría grave; en cuanto a deseo de estar con la gente, que también es un indicio de problemas interpersonales cuando se obtienen puntajes bajos, se evidencia que la población en estudio se concentra en su gran mayoría en las valoraciones positivas de esta dimensión, representado en un $84,9 \%$ dentro del total; es decir, la mayoría de los niños evaluados muestra deseo de estar con la gente, el $15,1 \%$ se ubica dentro del grupo que presenta síntomas de aislamiento social.
Otro de los ítems es hacer lo que dicen, con un $53,8 \%$ y problemas con los demás cuyo resultado arroja un $83,3 \%$. Lo anterior significa el $53.8 \%$ de los niños deja influir su comportamiento por los deseos de los demás, y el $16.7 \%$ reporta tener problemas con los demás.

En lo referente a la variable "autoestima negativa" definida por Kovacs(1992) como el valor negativo que se le da a la imagen y al concepto que se tiene de sí mismo y que se forja en relación con la información de las personas significativas y de las experiencias, agrupa dentro del presente estudio cinco ítems así: logros, en donde se visualiza que la población en estudio se encuentra en un nivel leve, aportando así el 45,1\% del total de la muestra, lo cual indica un nivel bajo de autoestima negativa; odio a sí mismo, con valoraciones menores, proporcionando un 92,3\%; ganas de morir, en donde la población de estudio se concentra en las valoraciones de respuesta bajas con un porcentaje de $62,4 \%$; autoimagen positiva, con un porcentaje de $66,1 \%$ indicando un nivel de autoestima negativa bajo dentro del grupo; sentirse bueno y sentirse querido, con un $91,9 \%$ y $84,8 \%$ respectivamente. Las puntuaciones bajas arrojadas por el presente estudio señalan que no existe valoración negativa de sí mismo dentro de la población estudia$\mathrm{da}$, pero no necesariamente indican autoestima positiva.

Todo lo anterior repercute drásticamente en la capacidad de adaptación del menor, dado que lo limita a nuevos aprendizajes de habilidades sociales y de solución de problemas. Además de afectar sus relaciones interpersonales con los demás miembros de la familia, especialmente los padres que; como lo reporta la literatura, no reconocen a tiempo estos síntomas y empiezan a tachar al menor como un rebelde o "hiperactivo", desviando la atención sobre el problema real; por ende estos menores no reciben el tratamiento adecuado para su diagnóstico, dando lugar a una cronificación y empeoramiento de los síntomas, lo que favorece entonces la aparición del riesgo de autolesionarse o no cuidarse. 
Detallando por puntos de corte y sexo se observa que $2.1 \%$ de los niños presentaron puntuaciones iguales a 17, y el $2.3 \%$ de las niñas este mismo valor. El $1.3 \%$ de la población puntúo con 18 donde los hombres obtienen el $1.6 \%$ y las mujeres el $1.0 \%$. De la población que mostró calificación de 19, el $1.6 \%$ son niños y el $1.5 \%$ son niñas. Dentro del punto de corte 20 , se ubica el $2.0 \%$ de la población general, discriminado en $1.6 \%$ para niños y $2.3 \%$ para niñas. Así mismo, el $2.1 \%$ de los hombres presentaron valores de 21 , y de igual forma el $0.8 \%$ de las mujeres presentaron el mismo valor. En el punto de corte 22 , se ubica el $0.8 \%$ de los niños y el $1.0 \%$ de las niñas. El $0.3 \%$ de los hombres obtuvo una puntuación igual a 23 , puntuación que no alcanzó ninguna mujer. Con puntuaciones iguales a 24 , estuvieron $1.1 \%$ de los hombres y $0.5 \%$ de las mujeres. El $0.3 \%$ de los varones y el $0.5 \%$ de las mujeres arrojaron puntuaciones iguales a 25 . Calificaciones de 27 y 28 fueron obtenidos en el $0.5 \%$ de los hombres, resultados que no mostró ninguna niña. El $0.3 \%$ tanto de las mujeres como de los hombres obtuvieron puntuaciones iguales a 29 con puntuaciones iguales a 30 , están el $0.5 \%$ de los hombres y el $0.3 \%$ de las mujeres. En el $0.3 \%$ de los niños y el $0.3 \%$ de las niñas se encontraron calificaciones de 36 y 37 , respectivamente.

Para el presente estudio se obtuvo una media de 10,52 y una desviación estándar de 5,6 un puntaje más alto que el encontrado en el estudio realizado por Gómez, Alviz y Sepúlveda (2003). en la ciudad de Medellín, en donde se alcanzó una media de 7,9 con una desviación estándar de 5,2, significativamente parecido al encontrado por la autora del CDI Kovacs (1992) que fue de 9,8. En otros estudios encontrados, se observa una media de 11,1.

Respecto a la fiabilidad del estudio debe destacarse que se obtuvo un coeficiente de consistencia interna aceptable (alfa de Cronbach de 0.77)

Los distintos estudios sobre el CDI están de acuerdo en su alta consistencia interna tanto en muestras clínicas (Kovacs, 1992;) como en normales (Davanzo y cols., 2004 ).
El estudio de Kovacs (1992) presentó un alfa de 0.86. Así mismo, Frías y cols., 1991 mostró 0.79 en alfa de Cronbach, resultado similar al obtenido por nuestro estudio. Otras publicaciones como la de Maria Kovacs con la población española (1992) y la de Gómez, Alviz Y Sepúlveda (2001) arrojan alfas de 0.81 y 0.84 respectivamente.

Como se puede observar, el Alfa de Cronbach alcanza en distintas investigaciones unos valores entre 0.79 y 0.94 . Estas cifras representan una gran homogeneidad considerando que se han obtenido con diferentes tipos de poblaciones y de tamaños de muestra, lo que señala la fuerte consistencia de los diferentes elementos de la prueba.

En nuestro estudio el CDI ha mostrado también una alta consistencia interna en su aplicación a la población escolar del municipio de La Ceja (Antioquia). En una muestra de 768 niños y niñas, pertenecientes a la población normal, el alfa de Cronbach obtenido es de 0.77 lo que parece ser un resultado adecuado y similar a los datos obtenidos en investigaciones anteriores.

\section{Referencias}

American Psychiatric Association (1994). Diagnostic and Estatistical Manual of Mental Disorders, fourth edition, DSM-IV, Washington.

Craighead,W., Curry, J., Ilardi,S(1995) Relationship of children depression inventory Psichological Assessment, 7,171-176.

Davanzo,P., Kerwin., Nikore, V.,Esparza, C.,Forness, S., Murelle,L. (2004). Spanish translation and reability testing of the child depression inventory. Child Psychiatry and humane development,35,75-92.

Del Barrio, V., Roa, ML., Olmedo, M., Colodron, F. (2002). Primera adaptación del CDI a la población española. Acción Psicológica, 3,263272. 
Doménech, E., Subira,S.,Comellas,MJ., Cuxart,E (1995).Children emotional disturbances in a sample of adolescents between 13-14 years. Atenas: 4 European Congress of Psychology.

Frias,D., Del Barrio,V., y Mestre,V (1991) Children Depresión Inventory. Evaluacion Psicológica, 7, 377-391.

Gómez, I. ,Alvis,A., Sepúlveda,MF(2003). Características psicométricas del instrumento. Children Depression Inventory (CDI) para detector sintomatología depresiva en niños de 8 a 12 años de la ciudad de Medellín. En: PSICOG (editores). Investigaciones de psicología clínica-cognitiva comportamental en la ciudad de Medellín: U. DE A.

Gómez, C. (2005). Psiquiatría y salud mental de niños y adolescentes: una necesidad. Revista Colombiana de Psiquiatría, 34 ,338-339.

Kashani, J.H. Y Schmid L.S. (1995). Epidemiología y etiología de los trastornos depresivos. En: M. Shaffi y R. Shaffi. La depresión en niños y adolescentes. Barcelona: Martínez Roca.

Kovacs, M. (1992). Children Depresión Inventory CDI (Manual). Toronto: multihealth systems.
Mantilla, LF., Sabalza, L., Ojeda, JM.,Caraballo, E., Campo, A., Diaz, LA (2003). Prevalencia de sintomatología depresiva en niños y niñas escolares de Bucaramanga. Trabajo presentado al 35 Congreso colombiano de Psiquiatría. Cali, 9-12 de octubre del 2003.

Ministerio de Protección Social (2004). El 40.1\% de la población entre 18 y 65 años ha tenido un trastorno mental en su vida. Boletín No 26,1-2.

Montero, I. y León, O. (2005). Sistema de clasificación del método en los informes de investigación en Psicología. International Journal of Clinical and Health Psychology, 5, 115127.

OMS (2003). Meeting on caring for children and adolescent with mental disorders: Setting WHO Directions. Geneve: WHO.

Riso, W. (1992). Depresión. Medellín: Centro de estudios avanzados en psicologia clínica.

Torres, Y. \& Montoya, I.D. (1997). II Estudio de salud mental y sustancias psicoactivas. Bogotá: Ministerio de salud. 